\title{
Validation of MRI Determination of the Penumbra by PET Measurements in Ischemic Stroke
}

\author{
Wolf-Dieter Heiss and Olivier Zaro Weber \\ Max Planck Institute for Metabolism Research, Cologne, Germany
}

The concept of the ischemic penumbra was formulated on the basis of animal experiments showing functional impairment and electrophysiologic disturbances with decreasing flow to the brain below defined values (the threshold for function) and irreversible tissue damage with blood supply further decreased (the threshold for infarction). The perfusion range between these thresholds was termed the "penumbra," and restitution of flow above the functional threshold was able to reverse the deficits without permanent damage. In further experiments, the dependency of the development of irreversible lesions on the interaction of the severity and the duration of critically reduced blood flow was established, proving that the lower the flow, the shorter the time for efficient reperfusion. As a consequence, infarction develops from the core of ischemia to the areas of less severe hypoperfusion. The translation of this experimental concept as the basis for the efficient treatment of stroke requires noninvasive methods with which regional flow and energy metabolism can be repeatedly investigated to demonstrate penumbra tissue, which can benefit from therapeutic interventions. PET allows the quantification of regional cerebral blood flow, the regional oxygen extraction fraction, and the regional metabolic rate for oxygen. With these variables, clear definitions of irreversible tissue damage and of critically hypoperfused but potentially salvageable tissue (i.e., the penumbra) in stroke patients can be achieved. However, PET is a research tool, and its complex logistics limit clinical routine applications. Perfusion-weighted or diffusion-weighted MRI is a widely applicable clinical tool, and the "mismatch" between perfusion-weighted and diffusion-weighted abnormalities serves as an indicator of the penumbra. However, comparative studies of perfusion-weighted or diffusionweighted MRI and PET have indicated overestimation of the core of irreversible infarction as well as of the penumbra by the MRI modalities. Some of these discrepancies can be explained by the nonselective application of relative perfusion thresholds, which might be improved by more complex analytic procedures. The heterogeneity of the MRI signatures used for the definition of the mismatch are also responsible for disappointing results in the application of perfusion-weighted or diffusion-weighted MRI to the selection of patients for clinical trials. As long as validation of the mismatch selection paradigm is lacking, the use of this paradigm as a surrogate marker of outcome is limited.

Key Words: penumbra; flow thresholds; core of infarction; neuronal activity; PET; PWI-DWI mismatch

J Nucl Med 2017; 58:187-193

DOI: 10.2967/jnumed.116.185975

Received Nov. 10, 2016; revision accepted Nov. 10, 2016.

For correspondence or reprints contact: Wolf-Dieter Heiss, Max Planck Institute for Neurological Research, Gleueler Strasse 50, 50931 Köln, Germany. E-mail: wdh@nf.mpg.de

Published online Nov. 22, 2016.

COPYRIGHT (C 2017 by the Society of Nuclear Medicine and Molecular Imaging.

\section{ENERGY REQUIREMENTS OF BRAIN TISSUE}

Because the energy demand of nervous tissue is very high, sufficient blood supply to the brain must be maintained consistently. The oxygen consumption of the brain is involved almost entirely in the oxidative metabolism of glucose which, in normal physiologic conditions, is the almost exclusive substrate for the energy metabolism of the brain (1). The glucose metabolized in neuronal cell bodies is used mainly to support cellular vegetative and housekeeping functions. Increases in glucose consumption (and regional blood flow) evoked by functional activation are confined to synapserich regions and are rather large compared with the demands of neuronal cell bodies (2).

Overall, $87 \%$ of the total energy consumed is required by signaling, mainly action potential propagation and postsynaptic ion fluxes, and only $13 \%$ is expended in maintaining membrane resting potential (3).

\section{FLOW THRESHOLDS FOR PRESERVATION OF FUNCTION AND MORPHOLOGIC INTEGRITY}

The different energy requirements for the maintenance of membrane function and for the propagation of information (signals) lead to different thresholds of energy consumption and, consequently, blood flow required for the supply of sufficient biochemical substrates as well as for the preservation of neuronal function and morphologic integrity.

Experimental work on the ischemic flow thresholds of brain tissue demonstrated the existence of 2 critical levels of decreased perfusion: first, a level representing the flow threshold for reversible functional failure (functional threshold); and second, a lower threshold below which irreversible membrane failure and morphologic damage occur. The range of perfusion values between those limits was called the "ischemic penumbra" (4), which was characterized by the potential for functional recovery without morphologic damage, provided that local blood flow could be reestablished at a sufficient level and within a certain time period. Whereas neuronal function is impaired immediately when blood flow drops below the functional threshold, the development of irreversible morphologic damage is time dependent.

The interaction of the severity and the duration of ischemia in the development of irreversible cell damage was studied by simultaneous recordings of cortical neuronal activity and local blood flow (5). On the basis of a large number of neurons assessed during and after ischemia of various degrees and durations, it was possible to construct a discriminant curve representing the worst possible constellations of residual blood flow and duration of ischemia still permitting neuronal recovery. Typical points on this curve 
are blood flow rates of almost 0,10 , and $15 \mathrm{~mL} / 100 \mathrm{~g} / \mathrm{min}$ maintained for periods of 25,40 , and $80 \mathrm{~min}$, respectively. Between 17 and $18 \mathrm{~mL} / 100 \mathrm{~g} / \mathrm{min}$, the duration of ischemia tends to be infinite, thus indicating that this low-flow state can lead to morphologic damage when maintained for very long, as-yet-undefined periods of time. These results broaden the concept of the ischemic penumbra: the potential for the postischemic recovery of functionally impaired cells is determined not only by the level of residual flow in the ischemic phase but also by the duration of the flow disturbance.

\section{PROGRESSION OF ISCHEMIC TISSUE DAMAGE}

Infarct progression can be differentiated into 3 phases. During the acute phase, at a flow below the threshold of energy metabolism required for the maintenance of basic housekeeping ( $\sim 20 \%$ of preocclusion values) in the core tissue, injury is a direct consequence of the ischemia-induced energy failure. The resulting terminal depolarization of cell membranes is established within a few minutes after the onset of ischemia. During the subsequent, subacute phase, the irreversible damage expands into the areas around the core, where flow ranges from $25 \%$ to $50 \%$ of preocclusion values (i.e., below the value required for function due to axonal and synaptic activities, defined as the penumbra), until—after several hours (usually $\sim 6 \mathrm{~h}$ )—the lesion has extended over the entire area with a critically reduced blood supply. Finally, a delayed phase of tissue injury evolves and may last for several days or even weeks; in this phase, secondary phenomena, such as vasogenic edema, inflammation, and programmed cell death, may contribute to further progression of tissue damage.

In the early phases of ischemia, reperfusion is a successful treatment that can prevent infarction if initiated when nerve cells are not irreversibly damaged. If residual flow is low or close to zero, the time for effective reperfusion is short, and treatment often cannot be initiated early enough. In the subacute phase, efficient reperfusion can be attained in many cases and is the basis for the

\section{NOTEWORTHY}

- The ischemic penumbra indicates brain tissue with perfusion decreased to a range between the threshold for reversible functional deficit and above the level for irreversible damage. The penumbra is the basis for the success of reperfusion therapy.

- In humans, the ischemic penumbra can be reliably determined only by quantitative measurements of regional cerebral blood flow and regional cerebral oxygen consumption.

- PW/DW MRI provides estimates of reduced regional perfusion and tissue damage. The mismatch between the obtained images is used as a surrogate marker of the penumbra.

- PW/DW MRI estimates of critically reduced perfusion and irreversible tissue damage should be validated by quantitative measurements for a reliable application of the mismatch concept.

- The use of nonvalidated PWI and DWI signatures for selection of patients has led to failure of several treatment trials in acute ischemic stroke. successful treatment of stroke by thrombolysis (6) or interventional thrombectomy (7).

Many electrical and biologic disturbances interact in the progression of irreversible cell damage in ischemia. Periinfarct spreading depression like depolarizations play a central role in the cascade of molecular mechanisms involved in the propagation of ischemic damage; they include the release of excitatory and inhibitory neurotransmitters, the activation of receptors and receptoroperated ion channels, the influx of calcium, free-radical formation, nitric oxide generation, dysfunction of the endoplasmic reticulum, mitochondrial disturbances, and others (8).

\section{IDENTIFICATION OF PENUMBRA BY IMAGING}

The concept of the penumbra is based on neurophysiologic and functional studies with experimental models of focal ischemia. The transfer of this concept to imaging modalities is difficult because most markers used in experimental studies necessitate invasive procedures. Monitoring pathophysiologic changes in humans requires noninvasive imaging modalities to provide quantitative maps of several important physiologic variables, including regional cerebral blood flow (CBF), regional cerebral blood volume, and regional cerebral metabolic rates for oxygen $\left(\mathrm{CMRO}_{2}\right)$ and for glucose. Up to now, only PET has been able to measure these variables repeatedly.

Early PET studies of stroke identified various tissue compartments within a brain territory compromised by ischemia (9-12). Tissue with $\mathrm{CBF}$ of less than $12 \mathrm{~mL} / 100 \mathrm{~g} / \mathrm{min}$ or $\mathrm{CMRO}_{2}$ of less than $65 \mu \mathrm{mol} / 100 \mathrm{~g} / \mathrm{min}$ at the time of measurement (usually several hours after stroke) was found to be infarcted on late CT scans. Relatively preserved $\mathrm{CMRO}_{2}$ was an indicator of maintained neuronal integrity in regions with severely reduced CBF. This pattern, coined "misery perfusion" (13), served as a definition of the penumbra, characterized as the area with an increased oxygen extraction fraction (OEF) (up to $>80 \%$ from the normal value of $\sim 40 \%$ ) (Fig. 1). Regions with CBF of 12-22 mL/100 g/min have an unstable metabolic situation; infarction might develop if low flow persists.

These PET studies (9-13) allowed the classification of 3 regions within the disturbed vascular territory: the core of ischemia, with a flow of less than $12 \mathrm{~mL} / 100 \mathrm{~g} / \mathrm{min}$, usually showing a transition to necrosis; a penumbra region, with a flow of 12-22 $\mathrm{mL} / 100 \mathrm{~g} / \mathrm{min}$, containing tissue that is still viable but that has uncertain chances for infarction or recovery; and an area of oligemia, with a flow of greater than $22 \mathrm{~mL} / 100 \mathrm{~g} / \mathrm{min}$, not

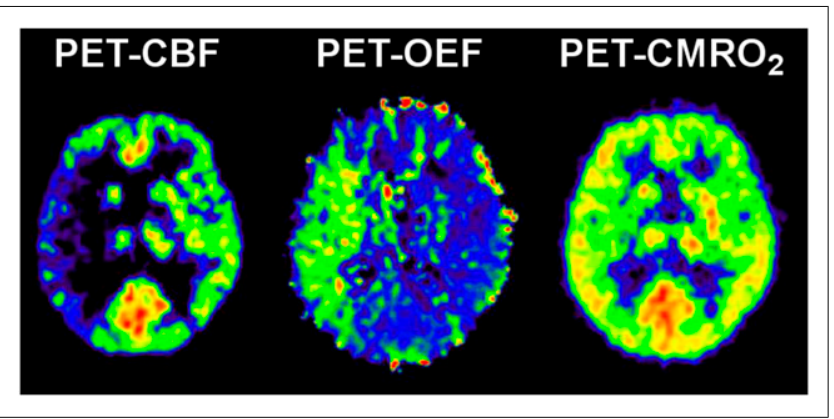

FIGURE 1. ${ }^{15} \mathrm{O} P E T$ measurement of $\mathrm{CBF}, \mathrm{OEF}$, and $\mathrm{CMRO}_{2}$ in patient with acute stroke. Penumbral tissue is represented by reduced $\mathrm{CBF}$, compensatory increased $\mathrm{OEF}$, but normal $\mathrm{CMRO}_{2}$. 
primarily damaged by the lack of blood supply. The condition of the tissue changes with time; the extent of the penumbra and its conversion to infarction is a dynamic process, and irreversible damage spreads from the core of ischemia to its border. This process can be monitored directly with advanced PET equipment; for example, changes in physiologic variables were studied after occlusion of the middle cerebral artery in baboons and cats. The experimental findings from sequential studies and anecdotal clinical investigations at different time points after the attack (14) implied that the extent of the penumbra, that is, of morphologically intact but functionally impaired tissue, depends on the time of measurement relative to the onset of ischemia. The volume is large and the flow values are low if the penumbra is defined in the initial hours of ischemia; at this point in time, reperfusion strategies are most effective. The volume is small if the penumbra is defined later, limiting the efficacy of treatment.

Measurement of blood flow values and determination of the OEF require arterial blood sampling, and clinical applicability is further limited by the complex logistics and instrumentation involved. Isolated flow determinations at a single time point might be confusing as long as the pattern over time is not known. A marker of neuronal integrity that can identify irreversibly damaged tissue irrespective of the time elapsed since the vascular attack and irrespective of variations in blood flow over time and that does not require arterial blood sampling is needed. The central benzodiazepine receptor ligand flumazenil binds to the $\gamma$-aminobutyric acid receptor, which is abundant in the cerebral cortex. These receptors are sensitive to ischemic damage and can therefore identify early neuronal loss (15).

\section{MRI MISMATCH AS SURROGATE FOR PENUMBRA}

Because of the complexity of the methodology, the limited access, the invasive and complicated procedures, and the exposure to radioactivity, PET cannot be applied in routine clinical settings. MRI studies of diffusion and perfusion might provide differentiation between the core and the penumbra: the early diffusionweighted imaging (DWI) lesion might define the ischemic core, and adjacent critically hypoperfused tissue might be identified by perfusion-weighted imaging (PWI) $(16,17)$. Therefore, brain regions with hypoperfusion assessed by PWI but with no or limited restricted diffusion (PWI-DWI mismatch) would be assumed to represent the penumbra.

This surrogate definition of the penumbra has several uncertainties (18). The initial diffusion lesion does not consist of only irreversibly infarcted tissue; diffusion lesions may be reversed if blood flow is restored at an early time point. However, the reversal of acute diffusion lesions beyond $3-4.5 \mathrm{~h}$ is infrequent (19). Critically hypoperfused tissue (i.e., the penumbra) cannot be clearly differentiated from tissue experiencing oligemia without the application of well-defined perfusion maps and thresholds (20); the PWI abnormality often overestimates the amount of tissue at risk of infarction. These facts are further accentuated by methodologic limitations because perfusion techniques and data evaluation techniques vary among centers $(21,22)$. Absolute or relative thresholds derived from PWI or DWI are still not reliable in predicting the fate of ischemic tissue, especially because of the difficulty in quantifying perfusion by MRI (23). With tracer bolus tracking, accurate measurement of the delay of tracer arrival, dispersion, and transit in pathologically perfused tissue and determination of the arterial input function (AIF) are difficult. These uncertainties are accentuated when collateral perfusion to areas beyond occluded arteries should be assessed. Differences in PET- and MRIbased CBF measures might also be due to the contrast agents-a nondiffusible intravascular agent (gadolinium-labeled diethylenetriaminepentaacetic acid) in perfusion MRI and a diffusible agent ( ${ }^{15} \mathrm{O}$-water) in PET (24).

\section{VALIDATION OF MRI SIGNATURES BY PET MEASUREMENTS}

A comparison of PWI or DWI results with quantitative measurements of flow values and oxygen consumption or flumazenil uptake in the same patients early after stroke is necessary for assessment of the accuracy of the applied signatures for predicting tissue outcomes. Several studies were performed to validate the mismatch as a surrogate of the penumbra in the discrimination of irreversibly damaged tissue, critically hypoperfused tissue considered at risk of infarction, and oligemic tissue considered not at risk.

\section{DWI of Ischemic Core}

The studies demonstrated that, compared with flumazenil PET, the DWI lesion predicted more or less the finally infarcted tissue but contained up to $25 \%$ false-positive, that is, surviving tissue (25). Other comparative studies of fully quantitative ${ }^{15} \mathrm{O}$ PET $\left(\mathrm{CBF}, \mathrm{OEF}\right.$, and $\mathrm{CMRO}_{2}$ ) in stroke showed that part of the DWI lesion had preserved normal $\mathrm{CMRO}_{2}$ and $\mathrm{OEF}$, as measured by PET (26-28). One study comparing PET-derived mean transit time with PWI-derived mean transit time suggested that this flow surrogate could improve infarct prediction within the DWI lesion (29). These studies as well as other studies indicated that parts of the DWI lesion showed an impairment of energy metabolism, which might involve penumbral tissue and therefore could be reversible. The high sensitivity but low specificity of DWI for infarct core detection might be improved if a threshold were applied to delineate the DWI lesion, for example, a relative intensity of $120 \%$ of the DWI lesion (25).

\section{PWI of Normal Perfusion}

Perfusion values determined by MRI bolus tracking were comparable to flow rates measured by ${ }^{15} \mathrm{O}-\mathrm{H}_{2} \mathrm{O}$ PET in normal volunteers, but tracer delay caused errors in CBF estimates, even in healthy people, and should be corrected (30).

\section{PWI of Penumbra}

The difficulty in defining the penumbra with the PWI-DWI mismatch is thought to be mainly related to PWI data acquisition, which is a complex process, and the surrogate parameters used to estimate perfusion are variable and somewhat arbitrary (31). Overall, PWI is unable to provide a reliable quantitative estimation of cerebral perfusion in stroke compared with gold standards such as PET, SPECT, or Xe CT (32-34).

Dynamic susceptibility contrast PWI relies on surrogates of perfusion parameters calculated from the nondeconvolved or deconvolved tissue residue function (time contrast curve) of the intravascular MRI contrast bolus. The nondeconvolved parameters, for example, time to peak (TTP) or first moment, are calculated without deconvolution or an AIF. The deconvolved parameters, for example, CBF, cerebral blood volume, mean transit time, and time to maximum (Tmax) 


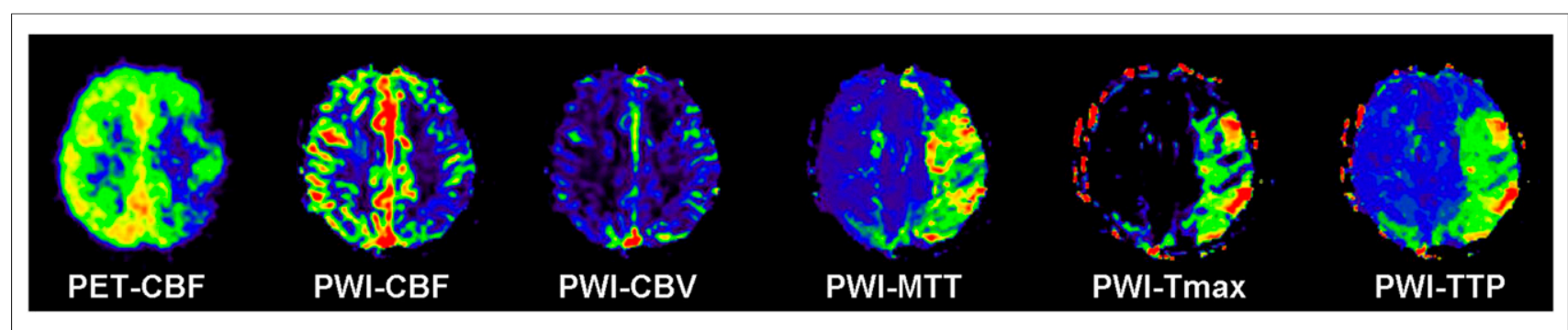

FIGURE 2. ${ }^{15} \mathrm{O}$-water PET measurement of $\mathrm{CBF}$ and $\mathrm{PWI}$ measurement of CBF, cerebral blood volume (CBV), mean transit time (MTT), Tmax, and TTP in patient with acute stroke. Areas of tissue at risk of infarction $(<20 \mathrm{~mL} / 100 \mathrm{~g} / \mathrm{min})$ are well depicted by PWI maps.

(Fig. 2) - which are thought to be more quantitative-rely on an AIF from large vessels (e.g., proximal middle cerebral artery) (31).

Several studies comparing PET and MRI have tried to validate the performance of perfusion maps from PWI in stroke patients. The goal of these comparative studies was the detection of the best parameter maps and the optimal thresholds for detecting tissue at risk of infarction (Fig. 3).

One study compared the nondeconvolved parameter TTP with PET-derived CBF. Delays of 4 and 6 s reliably identified hypoperfused tissue and excluded normally perfused tissue (the threshold was arbitrarily set to $20 \mathrm{~mL} / 100 \mathrm{~g} / \mathrm{min}$ ) but still overestimated the volume of critically hypoperfused (35) but salvageable tissue, that is, the penumbra. In another study (28), of 13 patients showing considerable PWI-DWI mismatch, only 8 had areas with elevated OEF typical of penumbra tissue, and these areas were larger on PWI-DWI than on PET. This overestimation of CBF by distribution maps was confirmed in another study comparing PET and MRI and involving 5 patients (32). A moderate correlation between PET-derived CBF and MRI-derived CBF was detected, and the correlation was even weaker when the data were pooled because of individual variations. However, the relative distributions of the perfusion maps were similar for PET and PWI. A comparative study of simultaneous ${ }^{15} \mathrm{O}-$ $\mathrm{H}_{2} \mathrm{O}$ PET and MRI in a clinical acute stroke setting (36) revealed

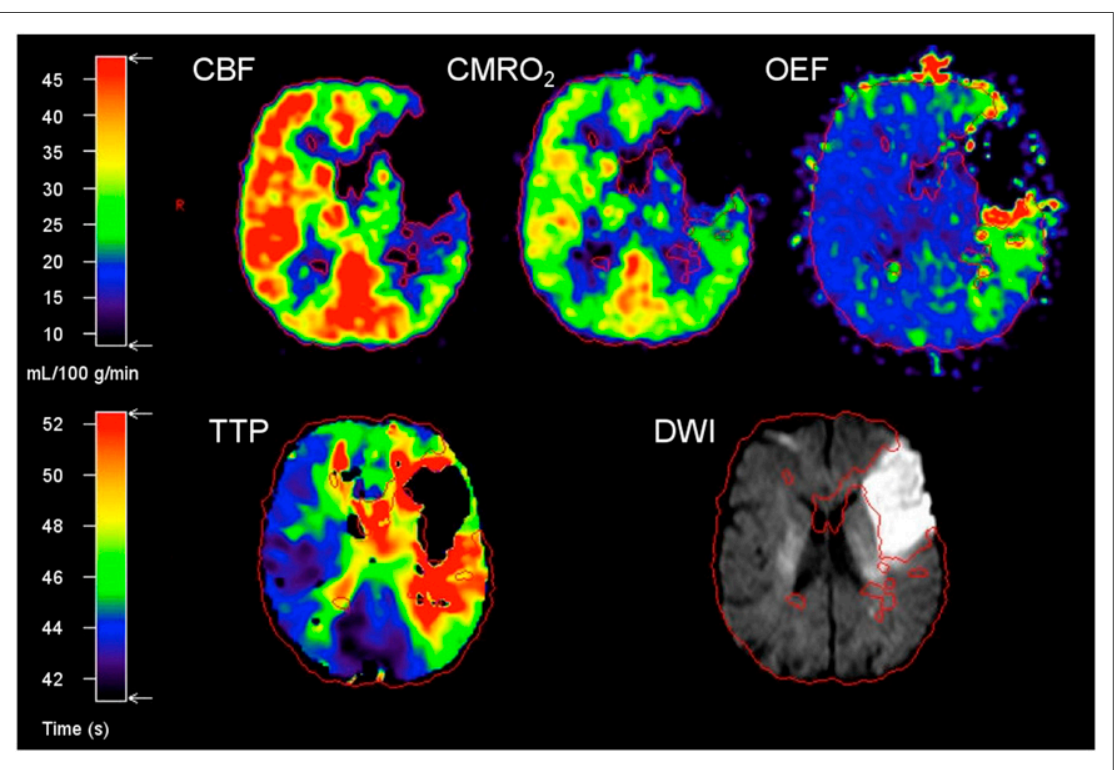

FIGURE 3. (Top) Identification of penumbra by hypoperfusion with preserved $\mathrm{CMRO}_{2}$ and elevated OEF $5 \mathrm{~h}$ after stroke. (Bottom) Corresponding mismatch of TTP and DWI. limited correspondence between PET-derived CBF and PWI-derived $\mathrm{CBF}$, TTP, and Tmax. Also, higher variability of PWI-derived CBF measures than of PET-derived CBF measures was detected. That study (36) revealed, in line with previous studies $(32,37)$, an overestimation of PWI-based CBF measures. Overall, the PWI-DWI mismatch volume, as conventionally calculated, does not reliably reflect misery perfusion - that is, the penumbra, as defined by PET.

Recently, several methods for improving the reliability of the assessment of perfusion with MRI methods were proposed (38-40), but they all need to be validated by quantitative measures. Therefore, PET-validated and -calibrated thresholds of the most predictive MRI perfusion maps must be implemented for optimal mismatch detection. More advanced analytic procedures may help to identify more reliably the threshold between critical hypoperfusion and noncritical hypoperfusion and to reduce the variance of determined values.

A comparative study of PET and MRI revealed the best thresholdindependent PWI maps as well as the optimal critical flow thresholds by comparative receiver-operating-characteristic curve analysis. That study established deconvolved maps for CBF $(<21.7 \mathrm{~mL} / 100 \mathrm{~g} / \mathrm{min})$ and time-driven maps for Tmax $(>5.5 \mathrm{~s})$ as well as a nondeconvolved map for regional TTP $(>4.2 \mathrm{~s})$ as the most predictive for discriminating the penumbral flow threshold, as defined by PET-derived $\mathrm{CBF}$, of less than $20 \mathrm{~mL} / 100 \mathrm{~g} / \mathrm{min}$ (41). These results were in line with those of the aforementioned study of 5 patients, which described the optimal penumbral flow threshold as a Tmax of greater than $5.4 \mathrm{~s}$ and a regional TTP of greater than $4.8 \mathrm{~s}$ (32).

Another study proposed a simple MRIbased and PET-validated calibration that reduced the variability of individual critical flow thresholds and consequently improved the detection of the mismatch, that is, the penumbra, in the acute stroke setting (42). Recent comparative studies of PET and MRI improved mismatch quantification and prediction of PWI maps by standardization of the placement of the AIF used for deconvolution (43) and establishment of the influence of deconvolution techniques, that is, standard singular value deconvolution and block-circulant singular value deconvolution (44). These studies recommended AIF placement within the proximal middle cerebral artery contralateral to the ischemic tissue and suggested 
that the standard singular value deconvolution method was adequate for calculating the best maps for mismatch detection (CBF and Tmax).

In summary, the improvement in mismatch detection achieved by these studies is important because current clinical studies, such as EXTEND and ECASS-4: ExTEND $(45,46)$, use PWI for treatment stratification and because standardization of postprocessing is crucial.

\section{PENUMBRA AS SURROGATE MARKER FOR TREATMENT EFFICACY}

The efficacy of treatment in ischemic stroke can be proven only in controlled randomized double-blind clinical trials. Because such controlled trials require large populations of patients from many stroke centers and, therefore, usually take a long time and require considerable funds, surrogate markers are used to predict potential therapeutic effects in small groups of patients. Proven effects on surrogate markers always must be confirmed in controlled trials with sufficient populations of patients. In recent years, the identification of salvageable tissue by neuroimaging has gained much interest as a surrogate marker for treatment efficacy in stroke.

The effect of the only approved conservative therapy for acute ischemic stroke was established in imaging studies in which reperfusion to penumbral tissue was followed by an improvement in neurologic deficits; reperfusion was significantly increased in recombinant tissue-type plasminogen activator-treated patients relative to controls (47). The volume of tissue salvaged by reperfusion was established in a study in which CBF determined by ${ }^{15} \mathrm{O}-\mathrm{H}_{2} \mathrm{O}$ PET within $3 \mathrm{~h}$ of stroke onset was compared with the volume of infarction determined by MRI $3 \mathrm{wk}$ after the ictus. That study demonstrated that a considerable portion of the critically hypoperfused tissue was probably salvaged by the reperfusion therapy.

The use of the PWI-DWI mismatch as the estimated zone of the penumbra has been proposed as a surrogate marker for the efficacy of stroke treatment (48). Several groups reported the results of serial PWI-DWI in patients after intravenous or intraarterial thrombolysis. Inhibition of lesion growth and even normalization of PWI (49) were seen with reperfusion after thrombolytic therapy, and the PWI-DWI mismatch was proposed as an effective selection criterion for recombinant tissue-type plasminogen activator treatment of patients admitted more than $3 \mathrm{~h}$ after the onset of symptoms (50). In some cases, perfusion deficits were resolved (51) and DWI signatures of early ischemic injury were reversed by prompt vessel recanalization (52). When a mismatch was still present 3-6 h after stroke onset, thrombolysis started beyond the accepted therapeutic window was followed by a favorable outcome $(53,54)$. As a consequence of the beneficial effect of thrombolysis in patients with a PWI-DWI mismatch, this signature was used for the selection of patients in several clinical trials (48).

Several studies included the selection of patients for intravenous thrombolysis on the basis of a PWI-DWI mismatch: in the DEFUSE (52) and EPITHET (55) studies, DWI and PWI volumes were calculated after patient enrollment, and outcomes were based on MRI profiles. The Desmoteplase in Acute Stroke (DIAS-2) (56) study included only patients with a visually assessed mismatch.

In the DEFUSE study (52), involving the open-label use of tissue plasminogen activator $3-6 \mathrm{~h}$ after symptom onset $(n=74)$,
40 patients had a mismatch (defined as a Tmax delay of more than 2 s) 1.2 times larger than the DWI lesion. Reperfusion and recanalization were associated with a favorable outcome in patients with a mismatch, and this effect was more apparent in the subgroup of patients who did not have a malignant profile. However, this study was not designed to show a clinical benefit because there was no placebo control group.

The EPITHET study (55) was a randomized double-blind placebo-controlled trial of tissue plasminogen activator used within 3-6 h after symptom onset $(n=101)$. With the same mismatch definition as that used in the DEFUSE study, the EPITHET study failed to demonstrate a statistically significant attenuation of infarct growth in the tissue plasminogen activator group. However, reperfusion was strongly associated with a good clinical outcome. Unfortunately, this study failed to prove mismatch imaging selection because of an insufficient number of patients who did not have a mismatch ("nonpenumbral" patients).

Desmoteplase, a newer thrombolytic agent, was administered 3-9 h after symptom onset in a multicenter placebo-controlled double-blind dose range study (DIAS-2; $n=186$ ) (56) of patients selected on the basis of a PWI-DWI mismatch $(20 \%)$ or a CT perfusion-based mismatch. This study did not show a benefit of desmoteplase. However, the results may have been affected by the selection criterion (visual assessment of mismatch), which may be inadequate for patient selection (57), and the small number of patients with vessel occlusion.

A metaanalysis of several mismatch-based thrombolysis studies of delayed treatment from the DIAS, DIAS-2, DEDAS, EPITHET, and DEFUSE trials revealed increased recanalization. However, this analysis did not confirm an improvement in clinical outcome with delayed thrombolysis (58).

Even though these trials did not show an improvement in clinical outcome, they supported the pathophysiologic basis of mismatch-based treatment selection. The missing confirmation of a clinical benefit in the selection of patients for treatment on the basis of MRI profiles may be related to an inappropriate definition of critically hypoperfused but salvageable tissue. A more complex analysis of data, including baseline DWI and PWI lesion volumes (59) and coregistration of mismatch and infarct locations (60), may be required.

The EXTEND trial and its European counterpart, the ECASS-4: ExTEND trial, both phase III trials, were designed to validate mismatch-based delayed treatment of ischemic stroke with recombinant tissue-type plasminogen activator $(46,47)$. These phase III, multicenter, randomized, doubleblind, placebo-controlled trials include 400 patients and evaluate recombinant tissue-type plasminogen activator versus a placebo within a 4.5- to 9-h time window and in wake-up strokes. Patient selection is based on a rigorous and standardized MRI and CT mismatch definition. However, as long as a validation of the mismatch selection paradigm used in these phase III trials is lacking, the selection of patients for delayed treatment on the basis of a mismatch cannot be recommended in routine care (58), and this surrogate marker of outcome must be used with caution $(61)$.

\section{DISCLOSURE}

No potential conflict of interest relevant to this article was reported. 


\section{REFERENCES}

1. Clarke DD, Sokoloff L. Circulation and energy metabolism of the brain. In: Siegel G, Agranoff B, Albers RW, Fisher S, eds. Basic Neurochemistry: Molecular, Cellular, and Medical Aspects. 6th ed. Philadelphia, PA: Lippincott-Raven; 1999:637-669.

2. Sokoloff L. Energetics of functional activation in neural tissues. Neurochem Res. 1999;24:321-329.

3. Laughlin SB, Attwell D. The metabolic cost of neural information: from fly eye to mammalian cortex. In: Frackowiak RSJ, Magistretti PJ, Shulman RG, et al., eds. Neuroenergetics: Relevance for Functional Brain Imaging. Strasbourg, France: Human Frontier Science Program; 2001:54-64.

4. Astrup J, Siesjö BK, Symon L. Thresholds in cerebral ischemia: the ischemic penumbra. Stroke. 1981;12:723-725.

5. Heiss WD, Rosner G. Functional recovery of cortical neurons as related to degree and duration of ischemia. Ann Neurol. 1983;14:294-301.

6. Hacke W, Kaste M, Bluhmki E, et al. Thrombolysis with alteplase 3 to 4.5 hours after acute ischemic stroke. N Engl J Med. 2008;359:1317-1329.

7. Menon BK, Campbell BC, Levi C, Goyal M. Role of imaging in current acute ischemic stroke workflow for endovascular therapy. Stroke. 2015;46:14531461.

8. Hossmann KA. Pathophysiology and therapy of experimental stroke. Cell Mol Neurobiol. 2006;26:1057-1083.

9. Ackerman RH, Correia JA, Alpert NM, et al. Positron imaging in ischemic stroke disease using compounds labeled with oxygen 15: initial results of clinicophysiologic correlations. Arch Neurol. 1981;38:537-543.

10. Baron JC, Bousser MG, Comar D, Soussaline F, Castaigne P. Noninvasive tomographic study of cerebral blood flow and oxygen metabolism in vivo: potentials, limitations, and clinical applications in cerebral ischemic disorders. Eur Neurol. 1981;20:273-284.

11. Lenzi GL, Frackowiak RSJ, Jones T. Cerebral oxygen metabolism and blood flow in human cerebral ischemic infarction. J Cereb Blood Flow Metab. 1982;2:321-335.

12. Powers WJ, Grubb RL Jr., Darriet D, Raichle ME. Cerebral blood flow and cerebral metabolic rate of oxygen requirements for cerebral function and viability in humans. J Cereb Blood Flow Metab. 1985;5:600-608.

13. Baron JC, Bousser MG, Rey A, Guillard A, Comar D, Castaigne P. Reversal of focal "misery-perfusion syndrome" by extra-intracranial arterial bypass in hemodynamic cerebral ischemia: a case study with ${ }^{15} \mathrm{O}$ positron emission tomography. Stroke. 1981;12:454-459.

14. Heiss WD, Huber M, Fink GR, et al. Progressive derangement of periinfarct viable tissue in ischemic stroke. J Cereb Blood Flow Metab. 1992;12:193203.

15. Heiss WD, Graf R, Fujita T, et al. Early detection of irreversibly damaged ischemic tissue by flumazenil positron emission tomography in cats. Stroke. 1997;28:2045-2051.

16. Baird AE, Benfield A, Schlaug G, et al. Enlargement of human cerebral ischemic lesion volumes measured by diffusion-weighted magnetic resonance imaging. Ann Neurol. 1997;41:581-589.

17. Barber PA, Darby DG, Desmond PM, et al. Prediction of stroke outcome with echoplanar perfusion-weighted and diffusion-weighted MRI. Neurology. 1998;51: $418-426$.

18. Kidwell CS, Alger JR, Saver JL. Beyond mismatch: evolving paradigms in imaging the ischemic penumbra with multimodal magnetic resonance imaging. Stroke. 2003;34:2729-2735.

19. Campbell BC, Purushotham A, Christensen S, et al. The infarct core is well represented by the acute diffusion lesion: sustained reversal is infrequent. J Cereb Blood Flow Metab. 2012;32:50-56.

20. Zaro-Weber O, Moeller-Hartmann W, Heiss W-D, Sobesky J. MRI perfusion maps in acute stroke validated with ${ }^{15} \mathrm{O}$-water positron emission tomography. Stroke. 2010;41:443-449.

21. Kane I, Carpenter T, Chappell F, et al. Comparison of 10 different magnetic resonance perfusion imaging processing methods in acute ischemic stroke: effect on lesion size, proportion of patients with diffusion/perfusion mismatch, clinical scores, and radiologic outcomes. Stroke. 2007;38:31583164.

22. Christensen S, Mouridsen K, Wu O, et al. Comparison of 10 perfusion MRI parameters in 97 sub-6-hour stroke patients using voxel-based receiver operating characteristics analysis. Stroke. 2009;40:2055-2061.

23. Calamante F, Gadian DG, Connelly A. Quantification of perfusion using bolus tracking magnetic resonance imaging in stroke: assumptions, limitations, and potential implications for clinical use. Stroke. 2002;33:1146-1151.
24. Raichle ME, Martin WR, Herscovitch P, Mintun MA, Markham J. Brain blood flow measured with intravenous $\mathrm{H}_{2}{ }^{15} \mathrm{O}$. II. Implementation and validation. $\mathrm{J} \mathrm{Nucl}$ Med. 1983;24:790-798.

25. Heiss WD, Sobesky J, Smekal U, et al. Probability of cortical infarction predicted by flumazenil binding and diffusion-weighted imaging signal intensity: a comparative positron emission tomography/magnetic resonance imaging study in early ischemic stroke. Stroke. 2004;35:1892-1898.

26. Guadagno JV, Warburton EA, Jones PS, et al. How affected is oxygen metabolism in DWI lesions? A combined acute stroke PET-MR study. Neurology. 2006;67:824-829.

27. Guadagno JV, Warburton EA, Jones PS, et al. The diffusion-weighted lesion in acute stroke: heterogeneous patterns of flow/metabolism uncoupling as assessed by quantitative positron emission tomography. Cerebrovasc Dis. 2005;19:239246.

28. Sobesky J, Weber OZ, Lehnhardt FG, et al. Does the mismatch match the penumbra? Magnetic resonance imaging and positron emission tomography in early ischemic stroke. Stroke. 2005;36:980-985.

29. Carrera E, Jones PS, Alawneh JA, et al. Predicting infarction within the diffusion-weighted imaging lesion: does the mean transit time have added value? Stroke. 2011;42:1602-1607.

30. Ostergaard L, Johannsen P, Host-Poulsen P, et al. Cerebral blood flow measurements by magnetic resonance imaging bolus tracking: comparison with $\left[{ }^{15} \mathrm{O}\right]$ $\mathrm{H}_{2} \mathrm{O}$ positron-emission-tomography in humans. J Cereb Blood Flow Metab. 1998;18:935-940.

31. Østergaard L. Principles of cerebral perfusion imaging by bolus tracking. J Magn Reson Imaging. 2005;22:710-717.

32. Takasawa M, Jones PS, Guadagno JV, et al. How reliable is perfusion MR in acute stroke? Validation and determination of the penumbra threshold against quantitative PET. Stroke. 2008;39:870-877.

33. Calamante F, Christensen S, Desmond PM, Ostergaard L, Davis SM, Connelly A. The physiological significance of the time-to-maximum (Tmax) parameter in perfusion MRI. Stroke. 2010;41:1169-1174.

34. Olivot JM, Mlynash M, Zaharchuk G, et al. Perfusion MRI (Tmax and MTT) correlation with xenon CT cerebral blood flow in stroke patients. Neurology. 2009;72:1140-1145.

35. Sobesky J, Zaro-Weber O, Lehnhardt FG, et al. Which time-to-peak threshold best identifies penumbral flow? A comparison of perfusion-weighted magnetic resonance imaging and positron emission tomography in acute ischemic stroke. Stroke. 2004;35:2843-2847.

36. Werner P, Saur D, Zeisig V, et al. Simultaneous PET/MRI in stroke: a case series. J Cereb Blood Flow Metab. 2015;35:1421-1425.

37. Zaro-Weber O, Moeller-Hartmann W, Heiss W-D, Sobesky J. The performance of MRI-based cerebral blood flow measurements in acute and subacute stroke compared with ${ }^{15} \mathrm{O}$-water positron emission tomography identification of penumbral flow. Stroke. 2009;40:2413-2421.

38. Shah MK, Shin W, Parikh VS, et al. Quantitative cerebral MR perfusion imaging: preliminary results in stroke. J Magn Reson Imaging. 2010;32:796-802.

39. Copen WA, Deipolyi AR, Schaefer PW, Schwamm LH, González RG, Wu O. Exposing hidden truncation-related errors in acute stroke perfusion imaging. AJNR. 2015;36:638-645.

40. Nael K, Meshksar A, Ellingson B, et al. Combined low-dose contrast-enhanced MR angiography and perfusion for acute ischemic stroke at 3T: a more efficient stroke protocol. AJNR. 2014;35:1078-1084.

41. Zaro-Weber O, Moeller-Hartmann W, Heiss W-D, Sobesky J. Maps of time to maximum and time to peak for mismatch definition in clinical stroke studies validated with positron emission tomography. Stroke. 2010;41:2817-2821.

42. Zaro-Weber O, Moeller-Hartmann W, Heiss W-D, Sobesky J. A simple positron emission tomography-based calibration for perfusion-weighted magnetic resonance maps to optimize penumbral flow detection in acute stroke. Stroke. 2010;41:1939-1945.

43. Zaro-Weber O, Moeller-Hartmann W, Heiss W-D, Sobesky J. Influence of the arterial input function on absolute and relative perfusion-weighted imaging penumbral flow detection: a validation with ${ }^{15} \mathrm{O}$-water positron emission tomography. Stroke. 2012;43:378-385.

44. Zaro-Weber O, Livne M, Martin SZ, et al. Comparison of the 2 most popular deconvolution techniques for the detection of penumbral flow in acute stroke. Stroke. 2015;46:2795-2799.

45. Ma H, Parsons MW, Christensen S, et al. A multicentre, randomized, double-blinded, placebo-controlled phase III study to investigate EXtending the time for Thrombolysis in Emergency Neurological Deficits (EXTEND). Int J Stroke. 2012;7:74-80.

46. Amiri H, Bluhmki E, Bendszus M, et al. European Cooperative Acute Stroke Study-4: extending the time for thrombolysis in emergency neurological deficits-ECASS-4: ExTEND. Int J Stroke. 2016;11:260-267. 
47. Heiss WD, Thiel A, Grond M, Graf R. Which targets are relevant for therapy of acute ischemic stroke? Stroke. 1999;30:1486-1489.

48. Donnan GA, Baron JC, Ma H, Davis SM. Penumbral selection of patients for trials of acute stroke therapy. Lancet Neurol. 2009;8:261-269.

49. Marks MP, Tong DC, Beaulieu C, Albers GW, de Crespigny A, Moseley ME. Evaluation of early reperfusion and IV tPA therapy using diffusion- and perfusion-weighted MRI. Neurology. 1999;52:1792-1798.

50. Röther J, Schellinger PD, Gass A, et al. Effect of intravenous thrombolysis on MRI parameters and functional outcome in acute stroke $<6$ hours. Stroke. 2002;33:2438-2445.

51. Kidwell CS, Saver JL, Mattiello J, et al. Thrombolytic reversal of acute human cerebral ischemic injury shown by diffusion/perfusion magnetic resonance imaging. Ann Neurol. 2000;47:462-469.

52. Olivot JM, Mlynash M, Thijs VN, et al. Relationships between cerebral perfusion and reversibility of acute diffusion lesions in DEFUSE: insights from RADAR. Stroke. 2009;40:1692-1697.

53. Schellinger PD, Thomalla G, Fiehler J, et al. MRI-based and CT-based thrombolytic therapy in acute stroke within and beyond established time windows: an analysis of 1210 patients. Stroke. 2007;38:2640-2645.

54. Thomalla G, Schwark C, Sobesky J, et al. Outcome and symptomatic bleeding complications of intravenous thrombolysis within 6 hours in MRI-selected stroke patients: comparison of a German multicenter study with the pooled data of ATLANTIS, ECASS, and NINDS tPA trials. Stroke. 2006;37:852-858.

55. Davis SM, Donnan GA, Parsons MW, et al. Effects of alteplase beyond $3 \mathrm{~h}$ after stroke in the Echoplanar Imaging Thrombolytic Evaluation Trial (EPITHET): a placebo-controlled randomised trial. Lancet Neurol. 2008;7:299-309.

56. Hacke W, Furlan AJ, Al-Rawi Y, et al. Intravenous desmoteplase in patients with acute ischaemic stroke selected by MRI perfusion-diffusion weighted imaging or perfusion CT (DIAS-2): a prospective, randomised, double-blind, placebocontrolled study. Lancet Neurol. 2009;8:141-150.

57. Campbell BC, Christensen S, Foster SJ, et al. Visual assessment of perfusiondiffusion mismatch is inadequate to select patients for thrombolysis. Cerebrovasc Dis. 2010;29:592-596.

58. Mishra NK, Albers GW, Davis SM, et al. Mismatch-based delayed thrombolysis: a meta-analysis. Stroke. 2010;41:e25-e33.

59. Parsons MW, Christensen S, McElduff $\mathrm{P}$, et al. Pretreatment diffusion- and perfusion-MR lesion volumes have a crucial influence on clinical response to stroke thrombolysis. J Cereb Blood Flow Metab. 2010;30:1214-1225.

60. Ogata T, Nagakane Y, Christensen S, et al. A topographic study of the evolution of the MR DWI/PWI mismatch pattern and its clinical impact: a study by the EPITHET and DEFUSE investigators. Stroke. 2011;42:1596-1601.

61. Wardlaw JM. Surrogate outcomes: a cautionary note. Stroke. 2009;40:10291031. 